\title{
Correction to: Derivation of a Linearised Elasticity Model from Singularly Perturbed Multiwell Energy Functionals
}

\author{
Roberto Alicandro, Gianni Dal Maso, Giuliano Lazzaroni, \\ \& Mariapia Palombaro
}

Communicated by I. FONSECA

\section{Correction to: Arch. Rational Mech. Anal. \\ https://doi.org/10.1007/s00205-018-1240-6}

The original version of this article unfortunately contained mistakes. Due to typesetting errors Table 1 was not correctly displayed and the $\square$ of Theorems 1.7(ii), 1.8(i), 1.8(ii), 1.10, 2.3 and 4.2 was missing.

The original article has been corrected. 


\section{ROBERTO Alicandro}

Dipartimento di Ingegneria Elettrica e dell'Informazione,

Università di Cassino e del Lazio meridionale,

via Di Biasio 43,

03043,

Cassino,

FR,

Italy.

e-mail: r.alicandro@unicas.it

and

Gianni Dal Maso

SISSA,Via Bonomea 265,

34136 ,

Trieste,

Italy.

e-mail: dalmaso@sissa.it

and

Giuliano LazZaroni

Dipartimento di Matematica e Applicazioni "Renato Caccioppoli",

Università degli Studi di Napoli Federico II, Via Cintia, Monte S. Angelo,

80126 ,

Naples,

Italy.

e-mail: giuliano.lazzaroni@sissa.it

and

Mariapia Palombaro

Department of Mathematics,

University of Sussex,

Pevensey 2 Building, Falmer Campus,

Brighton,

BN1 9QH,

United Kingdom

e-mail: M.Palombaro@sussex.ac.uk

Published online July 24, 2018

(c) Springer-Verlag GmbH Germany, part of Springer Nature (2018) 